\title{
Historia del tiempo presente en El Salvador y memoria oral
}

La memoria historica es bien corra en El Salvador. Por eso no se acumula experiencia ni tradición.

Ignacio Ellacuría

El pasado no puede, ni debe ser negado, sino asumido desde la práctica presente, con vista a la utopia que se quiere llevar a cabo.

Rodolfo Cardenal

\section{Presentación}

María Carmen García-Nieto París fue una gran amiga de la Universidad Centroamericana "José Simeón Cañas" y de El Salvador. Uno de sus sueños era crear un archivo oral de la historia de la izquierda en El Salvador. De hecho, dio los primeros pasos en ese sentido, coordinando la elaboración de una batería de preguntas que posibilitaran la recuperación de la memoria histórica de aquellos salvadoreños, mujeres y hombres, que se involucraron con el proyecto revolucionario en los años setenta y ochenta. Los resultados de ese trabajo pionero se guardan en un archivo que la propia María Carmen se encargó de entregar al cuidado del Centro de Información, Documentación y Apoyo a la Investigación (CIDAI) de la UCA. Ella, además, quería dejar por escrito no sólo sus intuiciones melodológicas sobre el estudio del "liempo presente" y la "memoria oral", sino avanzar en el análisis de la identidad y los valores de la izquierda desde 1979 a 1994. Lamentablemente, su muerte, acaecida a fines de 1997 tras una grave enfermedad, le impidió llevar a término estos propósitos. De sus estudios dedicados a El Salvador sólo pudo acabar la parte metodológica, no así la parte dedicada al análisis de los valores e identidad la izquierda. Por su valor intrínseco y como un homenaje póstumo a esa gran intelectual y mujer que fue María

- Directora del Fondo Documental de la Fundació Uropia Joan N. Garcia-Nieto, Estudis Socials del Baix Llobregat. 
Carmen García-Nieto París, hemos querido dar para su publicación la primera parte de su trabajo, así como el esquema de la segunda parte, esto último con el fin de incentivar a otros cientistas sociales del país a que continúen la labor iniciada por María Carmen.

\section{Introducción}

Las palabras de Ignacio Ellacuría y Rodolfo Cardenal, que encabezan este artículo, sintetizan las molivaciones y el contenido del mismo. Queremos reivindicar la necesidad y el deber de conocer la historia presente de El Salvador y su transmisión a las nuevas generaciones, como punto de partida de un El Salvador libre, justo, igualitario y democrático. Es algo que está en camino, desde la firma de los acuerdos de paz, pero algo, que todavía es una utopía, lejos de alcanzar, pero que puede y debe construirse.

El Salvador se encuentra en una de las coyunturas más interesantes y esperanzadoras de su historia. La historia es el arma' que nos permite recuperar el conocimiento de la realidad nacional salvadoreña reciente y actual, en un momento de cambio, a través de un proceso de transición y construcción de la democracia.

En la formación social salvadoreña hay una ideología y una práctica de izquierda que se ha ido generando y ha actuado desde los años treinta, y muy especialmente en la década de los ochenta, durante el período de la guerra civil. Pero, leamos las palabras de Ignacio Ellacuría que abren este artículo: "la memoria histórica es bien corta en El Salvador. Por eso no se acumula experiencia ni tradición"2. En este sentido consideramos urgente recuperar la memoria colectiva de la izquierda salvadoreña y de la población en su conjunto, en una década clave en la transformación de la realidad nacional.

Memoria colectiva es la que está vinculada y anclada en un grupo concreto, con el que comparte espacio y tiempo. En nuestro caso, la que pertenece a todos los hombres y mujeres de El Salvador en las décadas de los sesenta a los noventa, convencidos de que la historia la hacemos todos y todas, mujeres y hombres del campo y de la ciudad; analfabetos y universitarios; militantes y gentes que apoyan y acompañan.

Este fue el objetivo de dos cursos metodológicos organizados por los departamentos de filosofía y sociología, en los cuales, como objeto de estudio y trabajo de campo, se propuso el recuperar la memoria colectiva de la izquierda salvadoreña, como elemento clave de su identidad individual y colectiva y, a la vez, fundamento para la búsqueda y formulación del perfil de una nueva cultura política, que sea altemativa ideológica y práctica al neoliberalismo que tiende a dominar la sociedad salvadoreña. 
A lo largo de este artículo intentamos presentar, como diremos más abajo, lo que ha sido un trabajo colectivo realizado por quienes dirigimos el curso y los/as alumnos/as. Presentamos el modelo de una investigación de nuestra historia del tiempo presente utilizando como fuente principal y única la fuente oral. Planteamos problemas e hipólesis y los treinta y nueve documentos orales creados constituyen el inicio de un Archivo oral en la UCA.

\section{Tiempo presente y memoria oral}

Nos hallamos ante dos conceplos, dos terrenos polémicos en el campo de la historia. El tiempo presente se analiza en algunas ciencias sociales - sociología, antropología, lingüística, periodismo- centrándose, principalmente, en el hoy, en lo aclual y nada más. La historia introduce la relación con el pasado y el futuro. El tiempo adquiere una función dinámica y dialéctica en perspectiva y prospectiva. Ahora bien, el tiempo presente, reciente, inmediato, es visto con recelo por quienes se centran en el análisis de olros tiempos y per quienes no quieren recordarlo, por carecer, dicen ellos, de este tiempo reciente, inmediato, actual, presente, de perspectiva y de fuentes para su estudio.

Por otra parte, el recuerdo y la permanencia de los acontecimientos en la vida de las personas, de los grupos, de las comunidades es un hecho. Nos transmiten los acontecimientos, cómo sucedieron, el por qué, cómo se vivieron y se viven de cara al futuro. Nos dan a conocer la vida y la participación en el proceso histórico que marca la construcción de un país, de todos sus protagonistas y no sólo de quienes están en el poder. Todo ello nos permite hablar de la existencia de una memoria individual y de una memoria colectiva.

Recuerdo y memoria que no se rellejan ni constan en las fuentes tradicionales de la historia. El historiador/a debe acercarse y conocerlas a través de las fuentes orales. Ellas son el instrumento para la recuperación de la memoria oral. Ocupan, todavía, un espacio marginal en amplios sectores de la investigación histórica, y así son consideradas por la comunidad científica que dirige la construcción del discurso histórico. Se las mira, únicamente, como una técnica y/o una fuente subjetiva sin valor, contrapuesta a la denominada objetividad de olras fuentes documentales utilizadas "tradicionalmente".

Así pues, al hablar de tiempo presente surge, inmediatamente, un problema epistemológico: ¿puede y debe este tiempo presente ser objeto de historia? Nuestra respuesta categórica es afirmativa. Hoy día se habla de historia reciente, inmediata. Han surgido instituciones y revistas que se denominan con este nombre? Se organizan seminarios, aparecen publicaciones y se teoriza sobre el tiempo presente, frente a quienes contestan su validez científica.

Aceptando, pues, que puede y debe ser objeto de conocimiento histórico, un segundo aspecto es preguntar, ¿por qué se habla de tiempo presente diferencián- 
dolo de la contemporaneidad? Hay que aceptar su singularidad y especificidad dentro del marco general de la contemporaneidad, es decir, del período que tradicionalmente se califica, a grandes rasgos, como contemporáneo, los siglos $\mathrm{XIX}$ y XX. El tiempo presente constituye un campo científico y específico en varios aspectos. Vamos a fijarnos en dos de ellos: el marco cronológico y las fuentes.

Respecto a la cronología y periodización, el tiempo presente abarca un período de tiempo que se centra en lo que se denomina "expectativa de vida humana" (50,60, 70, 80, 90 años) según las regiones. Es un tiempo que se caracteriza por la presencia de testigos vivos. He aquí lo singular y específico del tiempo presente. Más aún, el historiador/historiadora, a su vez, ha sido también testigo vivo y protagonista de los acontecimientos que intenta historiar. Testigo e historiador/a son personas reales que poseen una memoria y unos recuerdos que condicionan, sin duda, el trabajo de investigación.

Testigos e hisloriadores/as son personajes históricos que intervienen, ambos, a su manera, en la construcción del discurso histórico. El investigador/a busca al testigo, lo elige, le pregunta. El testigo responde. Ambos crean el documento histórico.

Este tiempo presente, con sujetos vivos, presenta la dificultad de delimitar la frontera entre momento presente, actualidad e instante pasado. Al hacer historia no puede olvidarse la dialéctica entre pasado-presente-futuro ya que lo que define la historia es su relación con el pasado. Y siempre nos interesan todos los aspectos de la realidad, no con mero sentido arqueológico, sino con la finalidad de comprender en los hechos sociales, su encadenamiento y sus mecanismos, procurando que la experiencia de la historia y su conocimiento "forme parte de la conciencia de quienes son y serán protagonistas de la historia que se hace todos los días".

Pero hay más. Quienes hacemos o intentamos hacer historia del tiempo presente no podemos, como los historiadores/as de siglos anteriores, atrincheramos en un período de tiempo establecido y fijo para siempre: los siglos XIII, XVII, XIX e incluso la primera mitad del siglo XX. Estamos obligados a integrar continuamente secuencias cronológicas que suponen tensiones y dificultades. No puede establecerse una única cronología o periodización para todas las regiones, ni una periodización fija.

A modo de ejemplo, desde una perspectiva europea, se señala el año 1945 , final de la segunda guerra mundial, como punto inicial del tiempo presente. Desde nuestra perspectiva salvadoreña, 1932 es la coyuntura clave que desencadena todo el proceso histórico y de cambios hasta 1997. Es el acta de nacimiento de nuestro tiempo. Es un período en el que se tiene en cuenta lo que es una expectativa de vida, y en el que se da lo singular del tiempo presente, es decir, la presencia de sujetos vivos, tanto testigos como historiadores/as. 
Teniendo en cuenta cuanto se ha dicho, podemos acercamos a una definición que nos dice que

"Por historia del tiempo presente —reciente, del tiempo presente o próxima, conceptos todos ellos válidos - entendemos la posibilidad de análisis histórico de la realidad social vigente que comporta una coetaneidad entre la historia vivida y la escritura de esa misma historia y los propios historiadores"“.

O con palabras del historiador alemán, Lutz Niethammer, quien diferencia la historia contemporánea de la del tiempo presente, porque ésta se caracteriza

"por el hecho de investigar sucesos y estructuras con los que los contemporáneos tienen, todavía, una relación de dominio y experiencia inmediata"s.

En el intento de asociar pasado, presente y futuro, el historiador/a se halla inmerso, al igual que el testigo e informante, en el movimiento actual de la historia que amplía su campo y hace una lectura nueva de la realidad de nuestro tiempo. Se tratan de conocer las raíces próximas de lo que se vive, se investiga la identidad colectiva e individual con el deseo de crear y comprender el futuro.

El ayer, el hoy y el mañana se entrecruzan y entrelazan. La retrospectiva y la prospectiva están profundamente unidas. El historiador/a vive la confrontación con una historia en perpetuo movimiento, en perpetua progresión y en un perpetuo despertar.

No es fácil la tarea de hacer la historia del tiempo presente en El Salvador. La historia de una transición, de un proceso democrático que tiene sus raíces en golpes de Estado, represión, muertes y una guerra. Es una historia en construcción en la que el sujeto se interfiere con el conocimiento, el actor con la acción histórica y la memoria con la interpretación. No se define sólo por la cronología y el método, sino, sobre todo, por un aspecto que "obliga a reexaminar, las relaciones de la memoria y de la historia... La memoria es el problema histórico reciente, nuestro problema"t. En efecto, la memoria

“alimenta la historia, para servir al presente y al futuro. Se debe acluar de modo que la memoria colecliva sirva a la liberación y no a la servidumbre de los hombres"7.

Hacer la historia del tiempo presente en El Salvador es ejercer el derecho de la propia generación protagonista a preguntarse por el significado y el sentido de su propia acción histórica. Es deber de esta generación hacer esta historia y recuperar la memoria histórica, silenciada en su totalidad o fragmentariamente, en función de unos intereses políticos. Ningún científico social posterior podrá hacerlo. El tiempo presente lo encontramos, lo hemos vivido y lo vivimos.

Testigos e historiadores/as tienen unos recuerdos y una memoria. Esto obliga, como hemos dicho antes, a acudir a nuevas fuentes. Son las fuentes orales". Esto por varias razones: (1) por la presencia de testigos y sujetos del hecho que 
se analiza y que constituyen un rasgo específico de la historia del tiempo presente; (2) por la necesidad de recuperar la memoria de las mayorias; (3) por el conocimiento y comprensión, no sólo del dato histórico, sino de la experiencia vivida.

La fuente oral no es la voz espontánea de los/as testigos. "Es un método y una técnica para recoger un corpus de información histórica en forma oral registrado en cinta magnetofónica". Corpus de información histórica que, a partir de un planteamiento teórico y de unos objetivos concretos, en el marco de un proyecto de investigación, recogido y grabado, se convierte en fuente y documento histórico. Es fuente en cuanto grabada. Es técnica a través de la entrevista. Es método, mediante el cual podemos acercamos a los sin voz y a las mayorías. La fuente oral introduce la oralidad en una historia que debe hacerse bien, y exige, por tanto, la utilización de todas las fuentes requeridas por nuestro objeto de estudio.

La fuente oral exige una interaccion entre testigo e historiador/a, creación del documento histórico; presupuestos teóricos; un proyecto. Estos son los cuatro elementos necesarios para que la fuente oral sea un documento histórico.

No hay duda, que la fuente oral permite una concepción más amplia del pasado inmediato, de su elaboración sociocultural como historia y que su práctica revierte en la comprensión del proceso hislórico, en general, incluyendo en él a las mayorías.

\section{Metodología}

A partir de estos presupuestos teóricos, este artículo pretende únicamente, como ya se mencionó, presentar un pequeño trabajo de investigación colectivo realizado en el marco de dos cursos metodológicos (marzo-abril de 1994 y enero-febrero de 1995) organizados por los departamentos de filosofía y sociología. Su contenido, desde el punto de vista metodológico, pretendía ser una iniciación al trabajo del análisis histórico y social del tiempo presente utilizando como fuente principal la fuente oral.

En primer lugar, se impartieron unas clases teóricas y, en segundo lugar, se realizó colectivamente el trabajo de investigación en el que participaron los alumnos y alumnas y, con ellos también, la profesora doctora María Carmen GarcíaNieto y París, directora del curso, y la licenciada Montserrat Elwes y Alvarez, como ayudante.

\subsection{Los cursos}

La metodología utilizada en los dos cursos fue similar. Puede sintetizarse en los siguientes puntos, a través de los cuales queda plasmado el carácter teórico y práctico de los mismos: 
(1) Seminario que incluye la presentación de los presupuestos teóricos y metodológicos. Elección del objeto de estudio en la investigación: "La izquierda salvadoreña: identidad y valores, 1979-1994". Diseño del proyecto. Elaboración de la muestra y del cuestionario. Discusión del tipo de entrevista por realizar. (2) Trabajo de campo: realización de las entrevistas. Elaboración por parte de todos/ as de un informe sobre la entrevista, resumen de la misma y en algún caso transcripción completa. (3) Presentación, en varias sesiones de trabajo, de los informes y resúmenes. Análisis coleclivo de las entrevistas, contrastándolas con las hipótesis de trabajo. Elaboración de conclusiones que quedarán plasmadas en este artículo.

\subsection{El objeto de estudio y el marco cronológico}

El objelo de la investigación es el análisis de la izquierda salvadoreña en un momento de ruptura y cambio, de la dictadura cívico-militar a la democracia, que se desarrolla en dos fases: guerra civil (1979-1990) y proceso de paz (19901994). Se toma como punto de partida el año 1979 en el que se dan una serie de acontecimientos que aceleran las contradicciones y provocan la generalización de la guerra: surgen nuevas fuerzas de izquierda y se realiza el proceso de unificación de las mismas mediante la constitución del FMLN. Todo ello en medio de una situación de crisis política y social que desemboca en la guerra civil.

1980-1989. La guerra civil divide a la población salvadoreña. La izquierda lucha por una transformación socioeconómica y política que dé paso a una organización más justa, más igualitaria y más libre en El Salvador. A lo largo de la guerra, la clase dominante y la derecha se reestructuran y crean un partido, ARENA, que triunfa en las legislativas del 88 y en las presidenciales del 89. Ese año, 1989, se lleva a cabo la ofensiva hasta el tope por parte del FMLN. No cesa la represión por parte de los "escuadrones de la muerte", plasmada en los asesinatos de los jesuitas en la UCA.

1990-1994. Se aceleran las negociaciones entre la guerrilla y el gobierno hasta la firma del Acuerdo de Chapultepec y el proceso electoral de marzo de 1994 con el triunfo de ARENA. No se trata únicamente de recoger información y analizar unos acontecimientos, de describir y narrar lo que ocurre. El objelivo principal del esludio es profundizar en la realidad social, polílica y de guerra a través de la percepción y de la vida de quienes fueron sus prolagonistas y descubrir los factores, es decir, los mecanismos, contradicciones y conflictos que influyen en el proceso histórico.

\subsection{Hipótesis de trabajo}

La memoria individual se teje entre la red de la memoria compartida de la colectividad. Esta memoria compartida se hace real en los lugares comunes de la 
memoria colectiva, que son aquéllos en los que la memoria está selectivamente encamada y que, por voluntad de los hombres y el transcurrir del tiempo, han perdurado como símbolos destacados de la familia, de la colectividad o de la patria. Recuperar del olvido los lugares comunes de la memoria colectiva de la izquierda salvadoreña es recuperar su identidad y la de cada uno de sus miembros. Es hacer historia, pero la historia de todos y con todos.

Esta investigación pretende retomar el diálogo con un colectivo de personas para quienes el eje central de su vida ha sido la voluntad de cambiar las cosas hacia un mundo más justo, y la lucha contra la opresión, la injusticia y la desigualdad ha sido la razón de sus acluaciones.

En este diálogo, a través de su palabra, ellos y ellas explicarán cuáles son las razones fundamentales de su actuación, cómo actúan ahora, si se han cansado o no, qué modelo de sociedad pretenden, con qué instrumentos lo quieren conseguir, etc.

A lo largo de la guerra, en esta izquierda salvadoreña, se generan valores y formas de comportamiento que influyen también en la población. Nuestras preguntas son éstas: ¿surgen sólo en una situación de crisis?, ¿cuáles son?, ¿se han perdido o se mantienen?, ¿por qué? ¿Hay que recuperarlos como aporte para una nueva altemativa de izquierdas? En este sentido hay algo nuevo. Son las peculiares dificultades de la realidad salvadoreña en proceso de transformación democrálica en un marco neoliberal. El modelo, los fundamentos filosóficos y polílicos y las formas organizativas que hasla ahora parecían válidas se han esfumado y cuestionan el llamado "pensamiento de izquierda".

En concreto, las hipótesis propuestas son las siguientes: (1) A lo largo del período de la guerra civil, en gran parte de la población salvadoreña se desarrollaron: (a) el sentido de pertenencia: la persona tendió a superar su ámbito individual y pasó a sentirse "parte de", a estar "comprometida con", dispuesta a "asumir" una misión colectiva; (b) la conciencia de poseer intereses de carácter social: más allá del interés individual se tendió a reconocer intereses de clase o de grupo social. El interés individual se entendió subsumido a un interés de naturaleza superior y de carácter colectivo; (c) la sensación de poseer la fuerza: como integrante de una "fuerza social o política". La persona tendía a percibir la factibilidad del proyecto colectivo a partir del sentimiento de capacidad o potencia de realización del interés de carácter social. (d) Surgen nuevas formas de convivencia y organización social, económica y política: comunidades, cooperativas, etc.

(2) Paralelamente a este proceso se dio una reacción de los sectores más conservadores que entablaron, conscientemente, una lucha ideológica y represiva a muerte, desde posiciones de derecha, para tratar de anular las nuevas prácticas y los nuevos valores de carácter colectivo y solidario. Es la represión. Se 
entabló la lucha de lo individual y privado versus lo comunitario y colectivo, de la privatización versus la comunidad o la cooperativa, de la competencia versus la solidaridad o la cooperación. Las armas segaron en su raíz la nueva vida solidaria que brotaba en medio de la guerra.

(3) Las aclitudes hegemónicas en el período de transición oscilan entre el neoconservadurismo y el posmodernismo. Un común denominador es no poner en cuestión el sistema. Se acepla el capitalismo y más o menos se acepla el pragmatismo, el relativismo ético y valorativo.

Prima el llamado pensamiento débil. La política se desideologiza, y deja el campo libre a una nueva forma de hacer política entendida estrictamente como gestión. Ante esto se dan múltiples reacciones. Se mantienen todavía los que siguen creyendo, en la teoría y la práctica, que es necesario cambiar el sistema.

\subsection{La entrevista}

Teniendo presente el objeto de estudio y las hipótesis, se opló colectivamente por realizar el tipo de entrevista denominado historia de vida. ¿Por qué? Porque la historia de vida al ser una narración del conjunto de los acontecimientos de una existencia individual, tal como una persona los "rememora" a través del recuerdo, nos permitía penetrar, a través de las historias personales, en la realidad social salvadoreña, conocer los antecedentes, los por qués de una determinada actitud y de una toma de conciencia, etc., relacionar el presente que se analiza con el pasado y el futuro por el que se vive y lucha. A través de ellas se contrasta el tiempo cíclico personal con el tiempo político.

La historia de vida permite reinscribir la experiencia individual en el pasado común y da la posibilidad de "leer la sociedad" a través de una biografia. Pero, sobre todo, las vidas de mujeres y hombres, sus preocupaciones, sus trabajos, sus aficiones, que se mezclan, muchas veces, con la pobreza y el dolor, permiten realizar un estudio más profundo del momento conflictivo que les tocó vivir.

Quizá lo más importante de la utilización de las historias de vida en la investigación es que permilen relacionar lo individual y lo colectivo, lo público y lo privado, lo familiar y lo social, ya que se analiza lodo el marco de relaciones sociales y se convierle así en instrumento para llegar a la historia global, ya que se amplía el concepto y el campo de la historia de lo político a lo social en el más amplio sentido del término"'.

Lógicamente la historia de vida no es una entrevista cerrada, sino semidirigida/ abierta, con la ayuda de un cuestionario-guia. Se deja hablar, libremente, al informante, quien, con anterioridad, conoce el cuestionario y el proyecto. El cuestionario se elaboró y dividió en dos partes, claves, ambas, en una historia de vida. Dos partes, no plenamente diferenciadas, sino íntimamente entrelazadas, pero que exigen al investigador/a tener presentes muchas cuestiones, que si el/a 
informante no explicita, el/la investigador/a debe suscitarle haciendo preguntas. Estas dos partes son: (1) la historia del ciclo de vida, es decir, toda la vida personal, familiar y laboral, y (2) la historia de aspectos cualitativos de la actividad política-sindical-social-asociativa.

Para ayudar, tanto a la realización de la entrevista como para hacer el resumen y el análisis respecto al tiempo histórico, se consideró la coyuntura de la guerra como momento clave de ruptura y se establecieron tres momentos que condicionan la vida y la experiencia de los/as informantes: antes, durante y después de la guerra.

En nuestra investigación, a través de las historias personales, nos hemos acercado y dialogado con el contexto histórico, tanto el familiar como el de la organización, el del frente, el del exilio, el del refugio, el del retorno, el de un nuevo asentamiento, etc. Ha sido, realmente, un diálogo entre lo privado y lo público, lo individual y lo colectivo, que abre el camino para el estudio de: (1) La familia, que aparece como el eje principal que vertebra casi todas las trayectorias vitales analizadas y, factor, muchas veces, que impulsa al compromiso. La familia, en general, padece la desintegración, en tiempo de guerra, y el reencuentro, a veces difícil, al llegar la paz.

(2) La represión es el segundo eje que vertebra las vidas de nuestros/as prolagonistas, al generar "guindas", desplazamientos, exilio, la organización y la inserción en la guerrilla. (3) Las muertes y masacres se muestran como un tercer eje que impulsa y decide el compromiso organizativo y guenillero. (4) La organización política o social y los diferentes tipos de compromiso, ya sea como combatientes o como actividad polílica y social en la relaguardia.

(5) Los desplazamientos internos "guindeando", los refugios, el exilio y el retorno. (6) El retorno, es decir, la inserción en la vida civil del guerrillero, del político clandestino y combatiente, pero también de los grandes grupos de campesinos exiliados en Honduras y Nicaragua, principalmente, lo que supone un nuevo asentamiento, la toma de tierras, la construcción de una nueva organización en comunidades, etc. Nuevas ocupaciones y nuevo trabajo político, y también el desencanto. (7) La extrema pobreza de los años sesenta y setenta, que afecta a la mayoría de la población campesina. Esta situación injusta es la que moliva, mayoritariamente, al compromiso social, político y guerrillero, con el fin de cambiar el país.

Estos siete puntos sintetizan la riqueza de las historias de vida que contienen, al mismo tiempo, un corpus de información histórica sobre acontecimientos del proceso histórico de El Salvador desde los años treinta hasla el presente. 


\subsection{La muestra}

En la elaboración de la muestra se tuvieron presentes dos criterios: el estratégico (entrevistar a dirigentes y personas de la base) y la representatividad. No son excluyentes, sino que se entrecruzan y complementan, como puede verse en el modelo que se siguió al elaborar las muestras basadas, principalmente, en: la representatividad. Al buscar a las personas que debían ser entrevistadas se utilizaron diferentes redes: la amistad, la organización política, la comunidad, etc. Al buscarlas siempre estuvieron presentes unas variables que ayudaban a alcanzar la representatividad. Estas variables nos guiaron en las dos fases de la investigación.

Primera fase (1994): Comunidades. Las variables utilizadas fueron:

- Espacial: campesinas y urbano-marginales.

* Militancia: dirigentes y base.

* Sexo-género: hombres y mujeres.

* Edad: niños, jóvenes y adultos/as.

- Organizaciones: polílicas, movimiento popular, organizaciones de masas, movimientos sociales.

- Trabajo: agrícola, construcción, ama de casa, etc.

- Estudios: analfabetismo, básico, bachiller.

- Comunidad: de resistencia, repatriados, ex combatientes, etc.

- Ocupación acrual: trabajo, capacitación, ama de casa, dirigente comunal, etc.

En esta primera fase se realizaron 23 entrevistas que quedan reflejadas en el Cuadro 1 , en el que queda constancia de las distintas variables utilizadas.

\section{Segunda fase (1995): Dirigentes.}

En primer lugar, se tuvieron en cuenta los distintos sectores de militancia que incidieron en todo el proceso revolucionario. Destacan los siguientes:

- Militancia polftica: las distintas familias del FMLN.

- Sindicalismo: diferentes sindicatos.

- Movimiento campesino

- Cooperativas

- Derechos Humanos

- Movimiento intelectual

- Movimiento cristiano: Comunidades Eclesiales de Base, teólogos. 
* Comandancia guerrillera

En segundo lugar, al contaclar a los dirigentes de cada uno de estos sectores se tuvieron presentes, también, las siguientes variables:

- Sexo-género: hombres y mujeres.

* Edad: desde los años treinta a los sesenta.

* Estado civil: soltero/a, acompañado/a, viudo/a, separado/a.

* Estudios: desde el básico a la universidad.

* Militancia: política, social, cristiana, sindical, etc.

- Ocupación actual: política, profesional, sindical, etc.

- Ideología: cristiana, comunista, socialdemócrata, socialcristiana, izquierda.

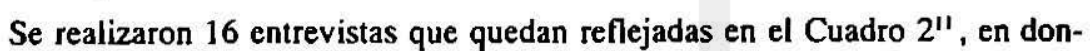
de aparecen todos los datos referidos a las trayectorias personales de los/as informantes.

El trabajo de campo y el análisis rápido de los documentos orales creados no puede considerarse una investigación acabada y de profundidad, que requiere, como ya se dijo antes, de la utilización no sólo de la fuente oral, sino de otras fuentes documentales, escritas y gráficas. La importancia, desde nuestro punto de vista, es que se presenta el modelo de una investigación con fuentes orales. Se plantean problemas e hipótesis que, los documentos orales creados, constituyen el inicio de un Archivo de la Palabra en la UCA. Con todo ello se quiere contribuir a hacer una historia, una sociología, una antropología con todos los hombres y todas las mujeres, a hacer un discurso social global, no parcial, y para todos $\mathrm{y}$ todas.

\subsection{Significado de la entrevista para los/as informantes y los/as entrevista- dores/as}

Para todos/as, informantes $y$ enlrevistadores/as el hecho de realizar una entrevista, con la finalidad de recuperar la memoria histórica de un período tan importante de la historia nacional, ha supuesto un hecho positivo.

Para los entrevistadores/as, en general, el realizar las entrevistas ha sido una experiencia iluminadora que les ha servido para conocer, más en profundidad y en verdad, la realidad nacional. Son expresivos estos testimonios:

"El impacto de regresar al campo después de muchos años y sobre todo a una zona ex conflictiva provocó ciertas inquietudes, comparar nuestra abundancia de recursos en nuestra ciudad y las limitaciones del agro, donde no hay energía eléctrica, agua polable, comunicaciones, elc... Personalmente toda la experiencia, ya sea antes, durante y después de la entrevista me ha afecla- 
do bastante al grado de provocar ciertas revaloraciones en relación con la realidad nacional"'2.

y el de quien:

"Ha aprendido que la historia de mi país la protagonizan los pobres; aquéllos que toman compromisos reales de transformar la realidad de este 'Pulgarcito de América'. Historia escrita con lágrimas, sudor y sangre. Historia escrita desde la montaña, la fábrica y los desvastados campos; escrita con estómagos vacíos y los pies en el lodo"13.

El trabajo de campo, en la creación de un documento oral

"Se ha convertido en fuente de riqueza y conocimiento al redescubrir de una forma latente, los valores y sufrimiento de este pueblo. Este trabajo ha cautivado mi atención, no solamente por su contenido histórico, sino también porque a la luz de él he descubierto el ansia de vivir y el dolor ante la muerte, por la cual este pueblo ha tenido que sobrevivir. Con solamente tener dos meses de residir en el país, este trabajo me abrió las puertas a escucharlo y a sentir su voz. Lo que había oído o leído con anterioridad de este tiempo de guerra en El Salvador, queda atrás, después de haber vivenciado los testimonios orales de los informantes"14.

Hubo, a veces, dificultades en la entrevista, no se recuerdan fechas, hay silencios, elc, pero

"esto viene a confirmar que una historia que no se escribe tiende a olvidarse. $Y$ es desde ahí que tenemos que comenzar a rescatar la memoria histórica de nuestros pueblos, quienes guardan una riqueza que sólo se encuentra hasta que se busca"'s.

Esta es la tarea y la función del investigador/a y la fuerza de la fuente oral para conocer y dar a la luz la verdadera historia de nuestros pueblos.

En general, todas las personas a las que se les pidió su colaboración, ya sea a través de contacto directo y personal por parte del entrevistador/a, en la primera fase de las entrevistas a las comunidades el año 1994, o bien por carta personal's firmada por los directores de los departamentos de filosofía y sociología, accedieron con gusto. El aspecto más importante que aparece en unas y en otras es el deseo de contribuir a que se sepa la verdad. Expresivas y con un sentido profundo de que la historia la hacemos todos son las palabras de D' Zoila:

"Ojalá que esta información le sirva y espero que al fin alguien escriba una historia de la realidad salvadoreña desde el pueblo. Pueden utilizar mi nombre si lo desean, eso ya no me importa con tal de que se sepa la verdad"'?.

A continuación de las palabras de $D^{2}$ Zoila podríamos añadir las de Fidel, "Saúl" y tantos otros. "Judith" accede a la entrevista y en sus palabras se perci- 
ben el compromiso y la responsabilidad de decir la verdad, pero al mismo tiempo se refleja todavía el temor a la represión:

"Si ya he nacido, debo de morir... Hemos sufrido bastante pero por lo menos estamos vivos, nosotros tenemos algo que contar"18.

Hasta aquí han sido testimonios de personas que están integradas en comunidades. He aqui el testimonio de Lorena Peña ("Rebeca"), comandante guerrillera y hoy dipulada por el FMLN:

"También te digo que la historia se empieza a escribir reciente, muy pocas páginas... Es útil rescalar testimonios para no perder la memoria frente a quienes quieren torcerla"14.

Una vez más puede afimarse que una historia que no se escribe tiende a olvidarse. $\mathrm{Y}$ es desde ahí que tenemos que comenzar a rescatar la memoria histórica de nuestros pueblos, quienes guardan una riqueza que sólo se encuentra cuando se busca.

Hay otros aspeclos que caracterizan la información obtenida en las entrevistas y que han sido importantes a la hora de analizar y oblener conclusiones. Estos son:

(1) La información obtenida no siempre es completa y explícita. En ocasiones, hay aspectos importantes y necesarios para valorar las motivaciones profundas que los llevan a la acción, que permanecen en la sombra (los silencios) o sólo son comentados por encima, lo que plantea algunos problemas - propios de los testimonios orales- que hemos tenido que valorar y confrontari"

(2) Por otra parte, el/la entrevistado/a no siempre se muestra consciente de los factores que le indujeron a involucrarse en acciones reivindicativas o sindicales $y$, con frecuencia, ofrece no sólo sus propias vivencias, sino que, además, incorpora como suyos, tal vez inconscienlemente, opiniones o discursos ajenos. Es necesario, por tanto, deslindar los testimonios directos de los indirectos, detectando los posibles estereotipos o prejuicios transmitidos por el informante. Como señala Niethammer "entrevistar al pueblo no siempre conduce a la verdad en la historia"2l, aunque en este caso tan válido es para nuestro estudio la veracidad de los datos facilitados por los testimonios, como la transmisión de las representaciones mentales que cada entrevistado/a se formó del período que le tocó vivir.

(3) Directamente relacionado con esta presencia de testimonios indirectos aparecen en el discurso oral las afloraciones de la llamada "memoria institucional" que, observa Joutard, procede no sólo del poder o de los grupos de la clase dominante, sino también de grupos u organismos no oficiales, marginados del poder, pero que generan su propia memoria oficial ${ }^{22}$. No hay duda que el discur- 
so del FMLN y de sus respectivas familias, en aquellos años, favoreció la constitución de una memoria institucional presente en algunos testimonios.

(4) Nuestras entrevistas ya son historia, porque el momento en que fueron recogidas forma parle de esa misma hisloria $y$, sin duda, hay que analizarlas teniendo presente la mediación del proceso electoral de 1994 y del inicio de la nueva democracia. Así, hay que entender la decepción, el desencanto y la esperanza en un futuro que no olvida los principios por los cuales se luchó militar, social y políticamente durante 12 años $^{22}$.

Cornellà de Llobregat, 23 octubre 1996.

\section{Notas}

1. Fraginals, Manuel Moreno, La historia como arma y otros estudios sobre esclavos $e$ ingenios, Barcelona: Crítica, 1983.

2. Ellacuría, lgnacio, "Análisis ético-político del proceso de diálogo en El Salvador", en Escritos Politicos, Tomo III, San Salvador: UCA Editores, 1991, p. 1393.

3. Institut d'Histoire du Temps Prèsent en París, que publica un boletín, es tal vez el más importante por sus estudios y publicaciones.

4. Cuesta, Josefina, Historia del presente, Madrid: Eudema, 1993, p. 11.

5. Niethammer, Lutz, "Para qué sirve la historia oral?", en Historia y Fuente Oral, No. 2, 1989, p. 13.

6. Pierre Nora del Institut d'Hisloire du Temps Présent de París.

7. Le Goff, J., El orden y la memoria. El tiempo como imaginario, Barcelona: Piados, 1991, pp. 178-183.

8. Cfr. Cuesta, Josefina, o.c., pp. 63-70 y Bédarida, François, "El tiempo presente, la memoria y el mito", en José Manucl Trujillano (ed.), Historia y Fuentes Orales; Memoria y sociedad en la España contemporánea, Avila, UNED-Avila Fundación Cultural Santa Teresa y Seminario de Fuentes orales, 1993, pp. 17-25.

9. El/a lector/a encontrará en el CIDAI los nombres de lodos y todas los/as participantes, pues allí se encuentran depositados los documentos orales y los análisis de los mismos.

10. Cfr. Ferrarolti, Franco, Histoire et histoires de vie. La méhode biographique dans les sciences sociales, Paris, Libraire des Meridiens, 1983; Bertaux, Daniel, "Los relatos de vida en el análisis social", en Historia y Fuente Oral, No. 1, 1989, pp. 87-96.

11. Para todo lo que se refiere a creación del documento oral, entrevisla, muestra, etc, consultar Folguera, Pilar, C6mo se hace historia oral, Madrid: Eudema, 1994.

12. Testimonio de Mario Elmer López, entrevista realizada el 19 de mayo de 1994.

13. Testimonio de Arley Martínez Guerra, entrevista realizada en la comunidad Guillermo Manuel Ungo, cantón Montepeque, Suchitoto, Cuscatlán, 18 de abril de 1994.

14. Palabras de Wilhelm Ignacio Lange Cruz, S.I. en el informe presentado de la entrevista realizada el 1 de abril de 1994.

15. Palabras de Héctor A. Estrella al comentar, en su informe, la entrevista realizada el 2 de abril de 1994.

16. De las cartas enviadas a dirigentes políticos, la respuesta de tres de ellas fue negativa.

17. Entrevistas realizadas el 28 de marzo y el 2 de abril de 1994. 
18. Entrevista realizada el 1 de abril de 1994.

19. Entrevista realizada el 23 de enero de 1995.

20. Cfr. Berg, Magnus, "La entrevista como mélodo de producción de conocimientos", Historia y Fuente Oral, No. 4, Barcelona, 1990, pp. 5-10; Vid. Fernández Benítez, Vicente, "Los lugares comunes de la memoria", en Trujillano, J.M., op. cit., pp. 7183.

21. Niethammer, Lutz, “¿Para qué sirve la Historia Oral?”, Historio y Fuente Oral, No. 2, Barcelona, 1989, pp. 3-26.

22. Cfr. Joutard, Philippe, Esas voces que nos llegan del pasado, México: FCE, 1986, pp. 253-296; Cfr. Portelli, Alessandro, "Historia y memoria: la muerte de Luigi Trastulli", Historia y Fuente Oral, No. 1, Barcelona, 1989, pp. 5-32.

23. En una segunda parte de este arlículo, que titularemos La izquierda salvadoreña: identidad y valores: 1979-1994, daremos a conocer los teslimonios directos de nuestros informantes. 